\title{
Two Layer 3D Floor Planning
}

\author{
Paul Horn* Gabor Lippner* \\ Department of Mathematics \\ Harvard University \\ Cambridge, MA, USA \\ \{phorn, lippner\}@math.harvard.edu
}

Submitted: Oct 17, 2012; Accepted: Nov 6, 2013; Published: Nov 15, 2013

Mathematics Subject Classifications: 05A16, 52C45, 68M07

\begin{abstract}
A 3D floorplan is a non-overlapping arrangement of blocks within a large box. Floor planning is a central notion in chip-design, and with recent advances in 3D integrated circuits, understanding 3D floorplans has become important. In this paper, we study so called mosaic 3D floorplans where the interior blocks partition the host box under a topological equivalence. We give representations which give an upper bound on the number of general 3D floorplans, and further consider the number of two layer mosaic floorplans. We prove that the number of two layer mosaic floorplans is $n^{(1+o(1)) n / 3}$. This contrasts with previous work which has studied 'corner free' mosaic floorplans, where the number is just exponential. The upper bound is by giving a representation, while the lower bound is a randomized construction.
\end{abstract}

\section{Introduction}

A (2D) floorplan is non-overlapping arrangement of rectangles within a larger host rectangle. It is a central notion in chip-design, where one wants to find an (in some sense) optimal arrangement of the components of a chip. The state-of-the-art methods for finding these optimal arrangements often involve enumerating through all possible arrangements, or generating arrangements randomly to find the optimal one. Hence finding efficient encodings for arrangements with a given number, $n$, of rectangles - and understanding the limitations of these - has become an important issue in the field of chip-design. Recently technology has enabled us to build chips in three dimensions [4]. This calls for an understanding of the $3 \mathrm{D}$ version of floorplans, where blocks are arranged within a host box. For clarity, as a matter of terminology, we refer to the largest outer box as a 'box', while we refer to the smaller inner components as 'blocks'.

${ }^{*}$ Research supported by grant FA9550-09-1-0090-DOD-35-CAP 
Mosaics are a special type of floorplans, where the smaller rectangles partition the host rectangle. Generic mosaic floorplans are such that they do not have adjacencies that could be removed by a small perturbation (the precise definition will be given in Section 2). Enumeration and efficient encoding of these is the first step towards understanding general floorplans. Obviously, there are uncountably many different (mosaic) floorplans. To meaningfully approach these questions one has to start with a notion of equivalence of floorplans, and enumerate and encode the resulting equivalence classes. We will consider the most widely studied notion, the so called topological equivalence (see Definition 10). The main problems are to find efficient encodings and asymptotic enumeration of topological equivalence classes of mosaic floorplans.

In the $2 \mathrm{D}$ case these problems have been studied extensively and satisfying answers have been found. The number of equivalence classes is essentially exponential in $n$. In fact they can be completely enumerated and their number turns out to be the number of Baxter permutations. There are nice bijections between floorplans and such permutations (see e.g. [2]), and also between floorplans and pairs of dual binary trees (see [7]) that provide efficient encodings. The crucial observation behind these results is always a certain kind of induction on the number of rectangles: if one removes the top left rectangle from an arrangement, the resulting gap can be filled by extending some of the other rectangles in the arrangement that were previously adjacent to the rectangle just removed.

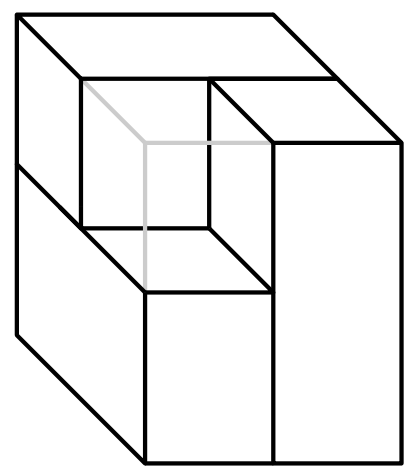

Figure 1: Diagonal Corner

This idea does not carry over to 3D floorplans. There is a certain kind of local arrangement (see Figure 1) where removing the small block in the front, neither of the adjacent blocks can be extended to cover the gap without creating overlaps. We shall refer to such an arrangement as a diagonal corner. This seems to be the only obstruction to generalizing the inductive argument: if one forbids diagonal corners, then a very similar induction works, see e.g. Chapter 3.3 of [1]. Hence many results carry over to 3D, in particular the number of such arrangements is again (up to lower order multiplicative terms) exponential. However the question is completely unresolved for general 3D arrangements. It was previously unknown even whether there exist more than exponentially many 3D floorplans. Indeed we are unaware of any non-trivial lower or upper bounds on the number of 
3D mosaic floorplans.

A number of other representations have been proposed for (often special classes of) 3D floorplans. A recent survey is given by [3]. This lists three attempts to represent mosaic floorplans. The 3D-corner block lists of Ma, Hong, Dong and Cheng [5] and OSequence of Ohta, Yamada, Kodama, and Fujiyosa [6] give representations for labeled floorplans without diagonal corners. Finally, there has been an attempt by Wang, Young, and Cheng [8] to generalize pairs of trees to encode 3D mosaic floorplans. Their approach would give an $n^{n}$ type upper bound, but their paper has errors and their encoding appears to be incomplete.

The starting point of our paper is nevertheless [8]. Let $\mathcal{F}_{n}$ denote the set of unlabeled generic 3D mosaic floorplans, and $\mathcal{T}_{n}$ the subset where there are only 2 layers in the $z$ direction. Our main contributions are the following.

- We give a non-trivial upper bound on the number of generic 3D floorplans.

\section{Theorem 1.}

$$
\log \left|\mathcal{F}_{n}\right| \leqslant 3 n \log n+O(n) .
$$

- We show that already the number of generic two-layer 3D mosaic floorplans up to topological equivalence is super-exponential. In particular using a random construction we prove

\section{Theorem 2.}

$$
\log \left|\mathcal{T}_{n}\right| \geqslant\left(\frac{1}{3}-o(1)\right) n \log n
$$

- Finally, exploiting duality of binary trees for 2D floorplans, careful analysis gives that this lower bound is asymptotically correct for 2-layer floorplans.

\section{Theorem 3.}

$$
\lim \frac{\log \left|\mathcal{T}_{n}\right|}{n \log n}=\frac{1}{3} .
$$

Remark 4. We find it rather surprising that already 2-layer floorplans have enough complexity to yield a super-exponential number of equivalence classes, especially in the light of the result that the number of general 3D plans without diagonal corners is only exponential. On the other hand we haven't been able to exploit multiple layers to construct significantly more floorplans. The best lower bound we have for $\log \left|\mathcal{F}_{n}\right|$ is, asymptotically, $\log \left|\mathcal{T}_{n}\right|$.

The paper is organized as follows. In Section 2 we give precise definitions of the various notions related to floorplans. We also recall the quaternary corner-tree construction of Wang et. al. from [8]. In Section 3 we give a random construction to prove Theorem 2. In Section 4 we show how 8-tuples of labeled corner-trees can be used to encode a 3D floorplan. Then an easy observation is used to reduce the required number of trees from 8 to 4 and finish the proof of Theorem 1. Finally in Section 5 we prove Theorem 3 by showing that in the case of 2-layer floorplans much less information is sufficient to encode the labelings on the trees. 


\section{Floorplans}

A mosaic floorplan is a subdivision of a large host box $H$ into $n$ smaller blocks $B_{1} \cup B_{2} \cup$ $\cdots \cup B_{n}=H$. A point in $H$ will be referred to as a corner if it is the vertex of at least one of the $B_{i}$ s.

Definition 5. The local configuration at a corner $x \in H$ is the following. Take a coordinate-parallel box $x \in L \subset H$ small enough that $L$ intersects exactly those blocks that contain $x$, but such that all these intersections have positive volume. These intersections then form a subdivision of $L$ into at most 8 blocks. This subdivision of $L$ is the local configuration at $x$, and $x$ is called the center of it.

Figure 2 shows examples of local configurations. We shall only be interested in certain special kinds of floorplans called generic floorplans.
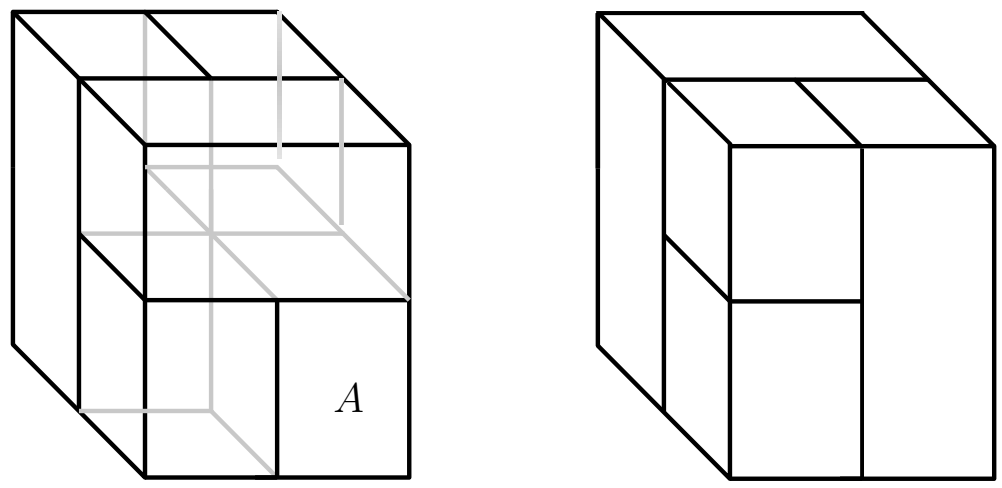

Figure 2: The corners in the middle of the cubes are the prototypes of generic corners. (The block labeled $A$ extends to the back of the configuration.)

\section{Definition 6.}

- A local configuration is generic if it satisfies the following condition:

- The center is the vertex of at most two of the blocks.

- If these two blocks share a face then there are at most 2 other blocks in the configuration. In this case the configuration is similar to the second example in Figure 2.

- Otherwise the two blocks may only intersect in a single point (the center) and there may be at most 3 other blocks in the configuration. In this case the configuration is similar to the first example in Figure 2

- A corner is generic if its local configuration is.

- A floorplan is generic if all its corners are. 
Remark 7. In two dimensions already the first of the three requirements defines what is a generic local configuration: The only thing we have to rule out is a corner where 4 rectangles meet.

Remark 8. There is a topological reason for the name 'generic'. Obviously, in the subdivision corresponding to a local configuration all the boxes contain the center. Non-generic local configurations are however not stable in this sense. They can be perturbed by an arbitrarily small change in the coordinates of the boxes into a new subdivision that doesn't have a unique center, because it contains disjoint boxes. This is not hard to see as there are only a few different local configurations to check. In fact it is also clear that one can choose the perturbation so that the resulting subdivision has only generic corners. Figure 3 shows a non-generic local configuration along with a perturbation that changes it.
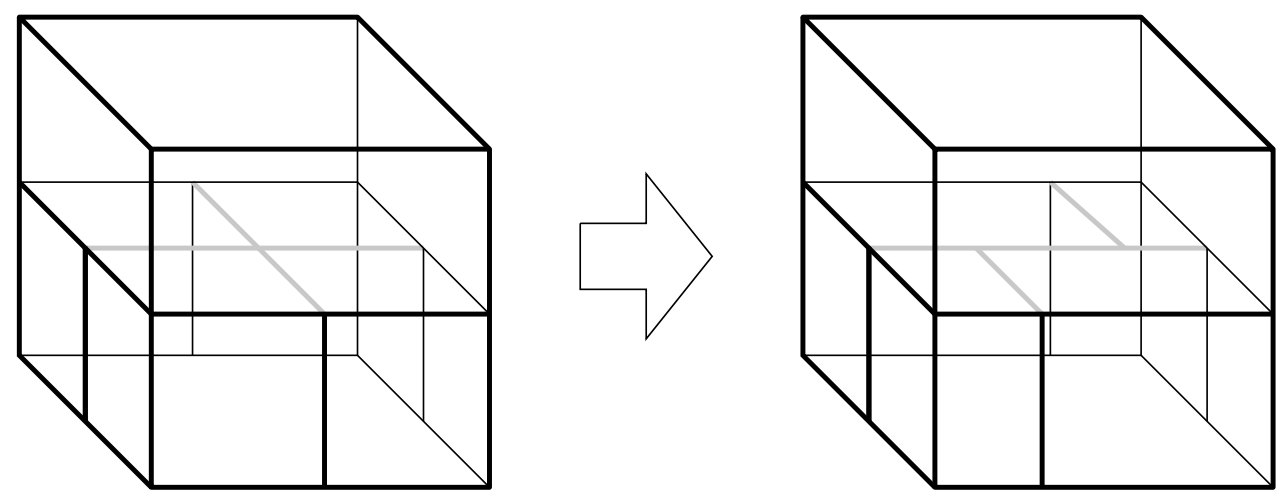

Figure 3: A non-generic configuration and its perturbation

There is a subtle point about generic corners that is important to keep in mind. We have seen that a non-generic local configuration can be perturbed into a generic one. One hopes that if the floorplan has a non-generic corner, then the whole floorplan can be slightly perturbed to get rid of this corner. This is the case in two dimensions. It is a known fact that a non-generic 2D floorplan can always be perturbed into a generic one. The same is not true however for 3D floorplans. Figure 4 shows a 3D floorplan which has two non-generic corners but that is globally rigid in the sense that any slightly perturbed floorplan will still have non-generic corners. Thus this notion of genericity is, in some sense, 'incorrect'. The main reason we still use this definition is because the properties of generic floorplans are much nicer compared to the non-generic ones.

\subsection{Dissection planes}

There is a classical encoding of floorplans which we now recall. One can reconstruct a floorplan given the appropriate coordinates of each face of each block. (The $x$-coordinate of the sides orthogonal to the $x$-axis, etc.) These coordinates cannot be arbitrary, however. When two blocks have touching faces, these sides obviously have to have the same 


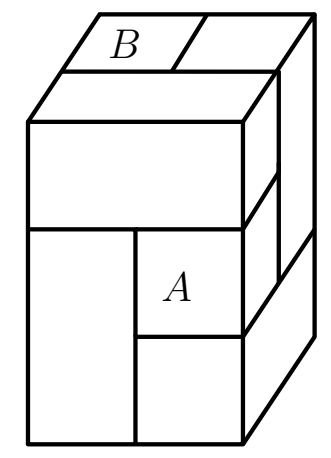

Figure 4: A globally rigid configuration where the interior corners of the block $A$ are non-generic. (The block labeled $B$ reaches all the way to the bottom.)

coordinate value. Hence it is enough to store one value for each group of faces that must have the same coordinate. This motivates the following approach.

\section{Definition 9.}

- A dissection plane (of a given floorplan) is the union of a maximal set of faces that must have the same coordinate value. More precisely let us say that two faces are attached if their intersection has positive area. This yields a graph on faces; dissection planes are the corresponding connected components of the graph.

- The dissection graph associated to a floorplan in a coordinate direction is a directed graph. Its vertices are the dissection planes orthogonal to the given direction. For each block of the floorplan one places an edge between the dissection planes containing the two faces of the block orthogonal to the given direction, and orients it towards the plane with the larger coordinate value.

Definition 10. Two floorplans are topologically equivalent if for each coordinate direction their corresponding dissection graphs are isomorphic. In the case of $3 D$-floorplans, this means that the $x$-, $y$ - and $z$-direction dissection graphs respectively must be isomorphic.

Remark 11. The name 'topological equivalence' comes from the following observation. Imagine that the walls of the blocks are mobile, as in a Japanese house. A deformation of a floorplan consists of translating a dissection plane in normal direction, creating no empty space and lengthening or shortening incident blocks in order to do such. It is easy to see that deformations preserve the dissection graphs.

It is a well known fact, however, (see, eg. [7] for a good explanation in the $2 D$ case) that the converse also holds: floorplans with isomorphic dissection graphs can be deformed into each other. Furthermore, given the dissection graphs, one can reconstruct the equivalence class of the floorplan as follows: The dissection graphs determine a partial order on the set of dissection planes. Extending this arbitrarily to a complete order, and 
assigning coordinate values respecting this order determines a choice for the coordinates of each block, hence one gets the desired floorplan.

The partial order setting makes the well known fact that we alluded to above more transparent. Consider two complete orders, both satisfying the partial order and differing by a transposition. In the floorplan setting this transposition corresponds to shifting one of the involved dissections planes past the other while maintaining the remaining order of the dissection planes. This is possible because the projected areas of the planes does not intersect - otherwise, these planes would be related in the partial order.

Definition 12. We will also use a one-dimensional analogue of dissection planes. Similarly to faces, we can say that two edges (of two blocks) are attached if their intersection has positive length. This yields a graph on edges, connected components in this graphs are going to be referred to as supporting segments. It is not hard to see (by looking at Figure 2) that, when two edges are attached, they lie in the intersection of two perpendicular dissection planes.

\subsection{Corner trees}

We shall need one more construction from [8] that will be used to encode floorplans, the so-called corner tree. Let us choose a corner of the host box and denote it by $P$. Each block $A$ in the floorplan has a corresponding corner, the one closest to $P$. If the corner is not $P$ itself, examining the possible shapes of a generic corner, it is not hard to see that there is always exactly one other block $B$ which shares this corner with $A$. Note that this will not be the corner of $B$ closest to $P$. Indeed, there are 4 possibilities for relative position of the second block with respect to the first block. They are either touching along a face in either of the three directions, or they intersect only in the corner itself. We say that, with respect to $P$, the block $B$ is the $(x / y / z / d)$-parent of $A$ in the four cases respectively (where $d$ stands for diagonal). Figure 7 shows the four types of adjacencies.

Definition 13. Suppose we are given a floorplan with the blocks labeled from 1 to $n$. For each corner $P$ of the host box, let $T_{P}$ be a tree whose vertices are the blocks, and each block is connected by an edge to its unique parent with respect to $P$. Vertices are labeled 1 to $n$ according to the numbers in the floorplan, and edges are labeled according to the type of the parent relation. The edge and vertex labeled tree constructed this way is called a corner tree of the floorplan. The tree has a unique root, the block that is adjacent to the corner $P$. It will also be useful to consider the corner tree without the vertex labels. This will be denoted by $\tilde{T}_{P}$.

It is easy to see that corner trees are invariant under deformations, hence topologically equivalent floorplans have isomorphic corner trees. Furthermore, every vertex in a corner tree can have at most four children, one for each type of edge. Thus a corner tree is a rooted edge-labeled quaternary tree on the set $\{1,2, \ldots, n\}$ with each vertex having at most one child for each of the four possible labels.

The labeling of the blocks does not make a huge difference in this construction. For each floorplan there are exactly $n$ ! ways to add the labels, hence enumerating labeled 
or unlabeled floorplans is equivalent. To encode labeled floorplans, we will use labeled corner trees. To encode unlabeled floorplans one can use the same set of trees, but with the labeling of the first tree arbitrary, and the labels are used only as an identification of vertices among trees. In other words, instead of considering eight trees with vertex labels, one can think of the eight trees given on the same vertex set, without any labels. This approach will be particularly useful when dealing with 2-layer floorplans.

In $2 \mathrm{D}$ it turns out that two corner trees corresponding to two opposite corners completely determine the dissection graphs of the floorplan, hence also its topological equivalence class. Furthermore, rather surprisingly, the vertex labeling of one tree can be reconstructed from the vertex labeling of the other tree, hence only one of the trees have to be vertex labeled. Since the blocks of a floorplan can be numbered arbitrarily, this means that 2D floorplans can be encoded with a pair of binary trees with only edge-labels. Since the number of (pairs of) binary trees is at most exponential in $n$, so is the number of floorplans. This is, however, not the case in 3D.

In [8] the authors claim that, analogously to the $2 \mathrm{D}$ case, a pair of (vertex labeled) corner trees corresponding to two opposite corners completely determine the dissection graphs of the floorplan. Since there are exponentially many pairs of quaternary trees, and $n$ ! identifications between vertices in the pair this would give an upper bound of the form $n ! \cdot \operatorname{expo}(n)=n^{(1+o(1)) n}$. However their proof is incomplete and we do not presently see if the gap can be filled.

\section{Lower bound construction}

In this section we prove Theorem 2 by constructing so many distinct floorplans. Consider an $N \times N$ grid, and $S \subseteq[N] \times[N]$. Construct a floorplan $F_{S}$ as follows:

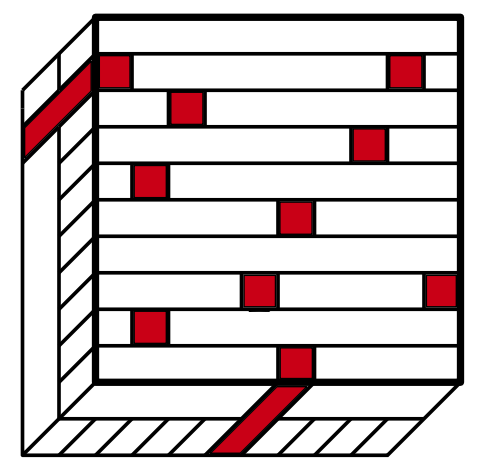

Figure 5: Vertical blocks in the positions of $S$

Place a height two block at each position in $S$. The remainder of the top level is filled in with horizontal strips, while the bottom level is filled in with vertical strips. For each set $S$, this yields a unique (though not necessarily generic) floorplan. Let us now count 
the total number of blocks. This floorplan has $|S|$ blocks of height 2 . If $|S|=0$, there are $N$ vertical and $N$ horizontal strips. Meanwhile, each element in $S$ which is not on the boundary of the grid, and not immediately adjacent to another element in $S$ adds an additional vertical and horizontal strip. We say that $S$ is good if this holds for every element in $S$. If $S$ is good, then there are $N+|S|$ horizontal and vertical strips, thus in total there are $2 N+3|S|$ blocks. If, in addition, there are no elements of $S$ that are diagonally incident then the floorplan $F_{S}$ is generic, thus $F_{S}$ represents an equivalence class in $\mathcal{T}_{2 N+3|S|}$.

It is clear that $S$ can be recovered from $F_{S}$. Thus the number of good $S$ where there are no diagonally incident elements gives a lower bound on $\left|\mathcal{T}_{n}\right|$.

Lemma 14. Fix $0 \leqslant t \leqslant \frac{(N-2)^{2}}{10}$ and $N$ sufficiently large. Let $S \subset\{2, \ldots, N-1\} \times$ $\{2, \ldots, N-1\}$ be a uniformly randomly chosen set of size $t$. Then

$$
\mathbb{P}\left(S \text { good and } F_{S} \text { generic }\right) \geqslant \exp \left(-50 \frac{t^{2}}{N^{2}}\right) .
$$

Proof. Choose $S$ to be a uniformly randomly chosen ordered set of size $t$. Let $S_{i}$ denote the first $i$ elements of $S$, and let $\mathcal{A}_{i}$ denote the event that $S_{i}$ is good, and $F_{S_{i}}$ is generic.

$$
\begin{aligned}
\mathbb{P}\left(\mathcal{A}_{t}\right) & =\mathbb{P}\left(\mathcal{A}_{1}\right) \mathbb{P}\left(\mathcal{A}_{2} \mid \mathcal{A}_{1}\right) \mathbb{P}\left(\mathcal{A}_{3} \mid \mathcal{A}_{2}\right) \cdots \mathbb{P}\left(\mathcal{A}_{t} \mid \mathcal{A}_{t-1}\right) \\
& \geqslant 1 \cdot\left(1-\frac{9}{(N-2)^{2}}\right) \cdot\left(1-\frac{18}{(N-2)^{2}}\right) \cdots\left(1-\frac{9(t-1)}{(N-2)^{2}}\right) \\
& \geqslant \exp \left(-\sum_{i=1}^{t-1} \frac{9 i}{(N-2)^{2}-9 i}\right) \\
& \geqslant \exp \left(-\sum_{i=1}^{t-1} \frac{9 i}{(N-2)^{2}-9 t}\right)=\exp \left(-\frac{9 t(t-1)}{2\left((N-2)^{2}-9 t\right)}\right) .
\end{aligned}
$$

Here, the first inequality comes from the fact that the first $i$ blocks forbid at most $9 i$ blocks for the $i+1$ st choice, and the second inequality comes from the real number inequality $\left(1-\frac{1}{x}\right) \geqslant e^{-\frac{1}{x-1}}$ for $x>1$. The result then follows noting that we used the fact that $t \leqslant \frac{(N-2)^{2}}{10}$ and the fact that $N$ is sufficiently large to somewhat simplify (1).

To complete the construction note that for a fixed $N$ and $t$, where $t<\frac{(N-2)^{2}}{10}$ and $n=2 N+3 t$, that

$$
\begin{aligned}
\left|\mathcal{T}_{n}\right| \geqslant \exp \left(-50 \frac{t^{2}}{N^{2}}\right)\left(\begin{array}{c}
(N-2)^{2} \\
t
\end{array}\right) & \geqslant \exp \left(-50 \frac{t^{2}}{N^{2}}\right)\left(\frac{(N-2)^{2}}{t}\right)^{t} \\
& =\exp \left(t \log \left(\frac{(N-2)^{2}}{t}\right)-50 \frac{t^{2}}{N^{2}}\right)
\end{aligned}
$$


Fix $t=N \log N$ and note that in this regime $n=(3+o(1)) t$ and $\log (n)=(1+$ $o(1)) \log \left(\frac{N}{\log N}\right)$. Using this in (2) gives the desired bound:

$$
\left|\log \mathcal{T}_{n}\right| \geqslant\left(\frac{1}{3}-o(1)\right) n \log n
$$

\section{Tree representation scheme}

In this section we prove Theorem 1 by giving a representation scheme for floorplans in $\mathcal{F}_{n}$. We do this in two steps. First we show that the dissection graphs of a floorplan can be reconstructed from the eight-tuple of corner trees $\left(T_{1}, \ldots, T_{8}\right)$ associated to the eight corners of the host box (see Figure 6 for the numbering of the corners). Then we show that knowing only $T_{1}, T_{3}, T_{6}$, and $T_{8}$, we can recover the four missing trees. The number of edge-labeled quaternary trees on $n$ vertices is exponential in $n$, hence this way we encode numbered floorplans with objects in a set of size at most $(n !)^{4} c^{n}$. But numbered floorplans are exactly $n$ ! as many as plain floorplans. Thus we get $\left|\mathcal{F}_{n}\right| \leqslant n !^{3} c^{n}$ or equivalently

$$
\log \left|\mathcal{F}_{n}\right| \leqslant 3 n \log n+O(n) .
$$

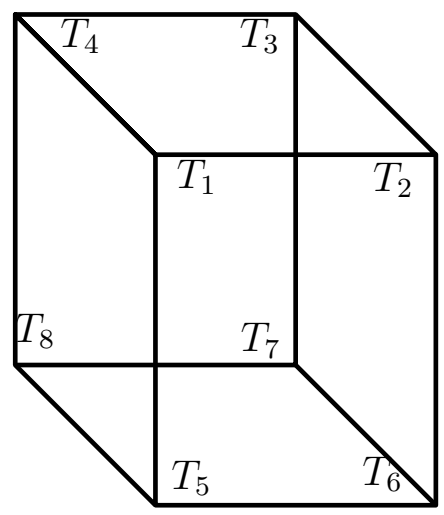

Figure 6: Corner/Tree identification

Proposition 15. The dissection graphs can be reconstructed from the corner trees $T_{1}, \ldots, T_{8}$.

Proof. The vertices of the dissection graphs are the dissection planes, and edges are given by the blocks. If we know the list of dissection planes, and which face of which block belongs to which dissection plane, then we know the graphs themselves. Hence it is enough to figure out which face is in which dissection plane. But since dissection planes are defined as unions of faces in an equivalence class, it is enough to recover these equivalence classes. Even though the equivalence classes were defined via the attachment relation, we cannot 


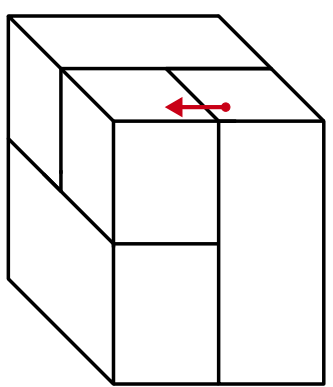

$x$-parent

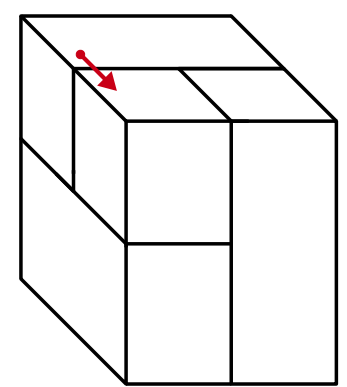

$y$-parent

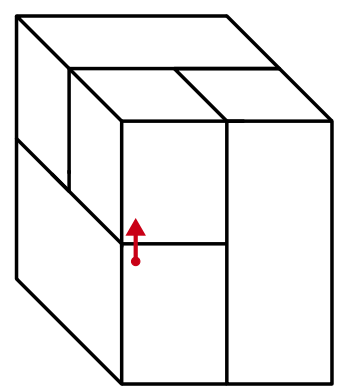

$z$-parent

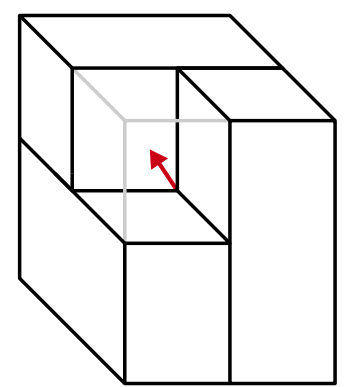

$d$-parent

In all cases, the block in the front, upper left is the parent and the source of the arrow is the child. In the case where the front block is a $d$-parent, the $d$-child is hidden behind the other block, and diagonally behind the front block.

Figure 7: Parent edge labels in $T_{1}$ tree

directly recover attachment from the list of corner trees. Instead, we shall use supporting segments (Definition 12) as an auxiliary tool.

First we shall show that, from the list of corner trees, one can recover which edges of which faces belong to the same supporting segment. This can be done by tracing a cycle of blocks around a supporting segment. Suppose we want to trace the segment that contains edge $X_{0} X_{1}$ of block $B_{0}$. (See Figure 8.) Then we look up $B_{0}$ in the tree corresponding to the $X_{1}$ corner, and find its parent $B_{1}$. Depending on what type of parent $B_{1}$ is, one of its edges, $X_{1} X_{2}$, will belong to the same supporting segment as $X_{0} X_{1}$. Then we take the tree corresponding to the $X_{2}$ corner of $B_{1}$, find the parent of $B_{1}$, and denote it by $B_{2}$. The edge $X_{2} X_{3}$ in $B_{2}$ then has to belong to the same supporting segment. When we reach the end of the segment, the process naturally 'turns around', and starts finding blocks backwards along the segment. Then eventually it hits the other end of the segment and starts coming back, until it finally hits $B_{0}$ again. At this point we have a complete list of blocks adjacent to the supporting segment. Also, for each block, we know which edge of it belongs to the segment. This whole process is depicted in Figure 8.

Now we are ready to recover dissection planes. Each dissection plane has two sides. By the definition of attachment, a dissection plane has connected interior. Thus, any face adjacent to one side can be reached from any other face adjacent to the same side by jumping from face to adjacent face. (Two faces are adjacent if they share an edge.) But when two faces are adjacent, their intersecting edges necessarily belong to the same supporting segment. Hence, starting from a face adjacent to a supporting segment, using the tracing method described above, one can find all other parallel faces that are adjacent to the same supporting segment. (And all such faces are necessarily contained in the same dissection plane!) Iterating this method, by connectivity, one eventually finds all faces on one side of a dissection plane. Finally the sides of a dissection plane can be connected together by tracing around a supporting segment on the boundary. 


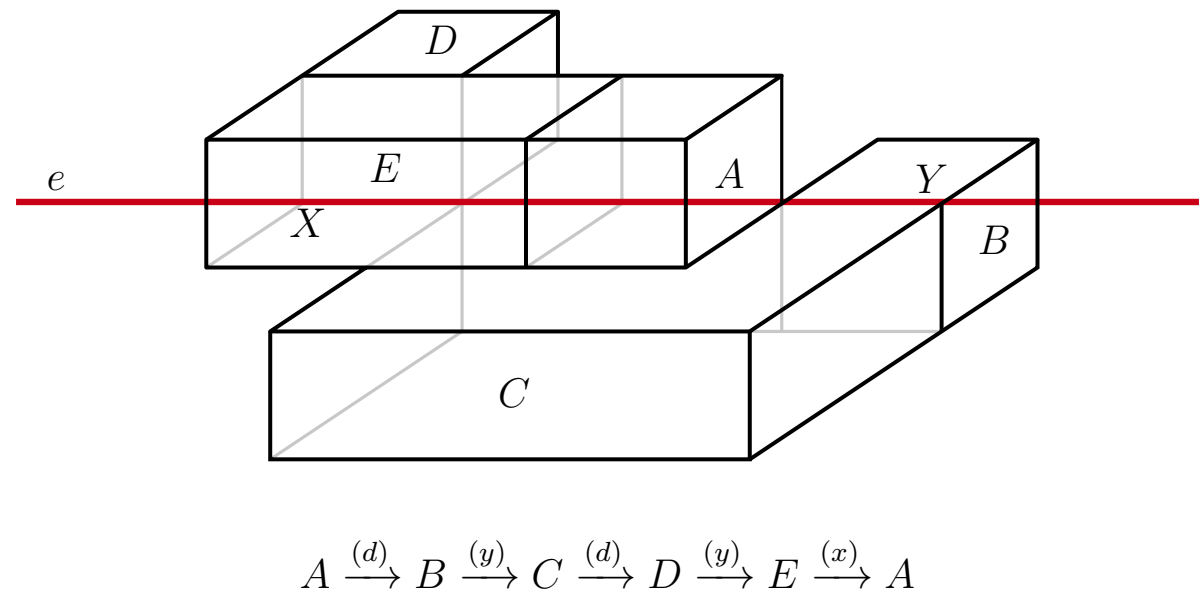

Figure 8: Tracing the supporting segment $e=X Y$

Proposition 16 ( 4 trees suffice). $T_{2}, T_{4}, T_{5}$ and $T_{7}$ are determined by $T_{1}, T_{3}, T_{6}$ and $T_{8}$.

Proof. The main observation is that there is a bijection between edges of the four unknown trees and the edges of the four given trees as follows. If there is a diagonal edge in $T_{1}$, say block $A$ is the diagonal parent of block $B$, then clearly there is a diagonal edge in $T_{7}$, namely block $B$ is the diagonal parent of block $A$ in that tree. And vice versa. Similarly, by looking at figure 7 one can see that if and only if $A$ is an $(x / y / z)$-parent of $B$ in $T_{1}$, if and only if is then $B$ an $(x / y / z)$-parent of $A$ in $T_{2} / T_{4} / T_{5}$ respectively. Hence each edge in a tree can be recovered from the trees corresponding to the three adjacent vertices and the one opposite vertex. This proves the proposition, and also completes the proof of Theorem 1.

\section{Label reconstruction}

In this section we prove Theorem 3. Given a two layer floorplan $F$, we know that it can be reconstructed from its eight labeled corner trees. The trees themselves can only be of exponentially many types, however the labeling is too expensive for our purposes. We have already seen that instead of vertex labels what we really need is bijections between the vertex sets of the trees. Even using Proposition 16 we would need three such bijections, and that is way more data than the $n^{n / 3}$ bound we are aiming for. The solution is that instead of recording all the identifications between vertex sets, we will only record partial identifications, and use intrinsic geometric constraints of the floorplan to recover the missing identifications.

So let us consider the unlabeled (or, rather, only edge-labeled) versions of our corner trees $\tilde{T}_{1}, \ldots, \tilde{T}_{8}$ associated to the two-level floorplan $F$. The two layers will be in the $z$ direction. We will refer to the two layers as the top and the bottom. Corners 1,2,3,4 are 
on the top, $5,6,7,8$ are on the bottom.

Definition 17 (Identification set). The full identification of a floorplan $F$ is the set

$$
\begin{aligned}
\mathcal{F} \mathcal{I}=\{(x, y): & x \in V\left(\tilde{T}_{i}\right), y \in V\left(\tilde{T}_{j}\right) \\
& \text { where } x \text { and } y \text { represent the same block of } F \text { in two different trees }\}
\end{aligned}
$$

An identification set is any subset $\mathcal{I} \subset \mathcal{F} \mathcal{I}$. An identification set $\mathcal{I}$ is strong if, together with the eight unlabeled trees, it determines $\mathcal{F} \mathcal{I}$.

Our goal is then to find strong identification sets as small as possible. Theorem 2 shows that any strong identification set has to be of size at least $n / 3$ asymptotically. We are going to show that for two-layer floorplans this can be achieved.

This will complete the proof of Theorem 3, by giving an injection from floorplans into the space of 8-tuples of $\{x, y, z, d\}$-edge labeled quaternary trees and strong identification sets of size $n / 3$. Note that a identification set consists of pairs $(x, y)$ so that no $x$ and no $y$ is in more than 8 pairs. Therefore the number of identification set of size $t$ is given by a two (multi)sets of size $t$ and a bijection between them. The number of such possibilities is at most

$$
\operatorname{expo}(n) \times(n / 3) !=n^{(1+o(1)) n / 3} .
$$

Since the number of $\{x, y, z, d\}$-edge labeled quaternary trees is also exponential, we will have given an injection into a space of size $n^{(1+o(1)) n / 3}$, which will complete the proof.

First we need some notation. Blocks of a floorplan $F \in \mathcal{T}_{n}$ can be broken into three classes, $T, B, D$ (as in top, bottom, double), denoting the blocks occupying only the top, only the bottom, or both layers. For a block $x \in F$ let $x_{i} \in \tilde{T}_{i}(i=1, \ldots, 8)$ denote the vertex in $\tilde{T}_{i}$ which represents $x$. It is in general impossible, given an $x_{i} \in \tilde{T}_{i}$ to determine $x_{j} \in \tilde{T}_{j}$ only knowing the unlabeled trees. However certain elements of $\mathcal{F} \mathcal{I}$ can be automatically reconstructed, as the following proposition shows.

Proposition 18. If $x \in T \cup D$, and $i, i^{\prime} \in\{1,2,3,4\}, x_{i}$ can be determined from $x_{i}^{\prime}$. Likewise, if $x \in B \cup D$, and $i, i^{\prime} \in\{5,6,7,8\}$ then $x_{i}$ can be determined from $x_{i}^{\prime}$.

Proof. The top and bottom planes are 2D floorplans with blocks from $T \cup D$ and $B \cup D$ respectively. The rooted subtree of $\tilde{T}_{i}, 1 \leqslant i \leqslant 4$ obtained by deleting all $z$-and $d$-edges are precisely the corner trees associated the corners of this $2 \mathrm{D}$ floorplan. We know from [7] that a 2D floorplan can be reconstructed from two of its corner trees corresponding to opposite corners. Furthermore, these trees do not have to be vertex-labeled. The bijection between their vertex sets can be recovered from the tree structure and edge-labeling. Hence looking at the subtrees obtained from $\tilde{T}_{1}$ and $\tilde{T}_{3}$ one can find the identification between the vertices in these two subtrees. Then the $2 \mathrm{D}$ floorplan can be reconstructed (up to topological equivalence). Finally, since the appropriate subtrees of $\tilde{T}_{2}$ and $\tilde{T}_{4}$ are corner trees of the same 2D floorplan, their vertices can be identified with the blocks in this floorplan, and hence to the vertices of $\tilde{T}_{1}$ and $\tilde{T}_{3}$. The second part of the proposition follows identically. 
For the remainder, we prove the following:

Proposition 19. There exists a strong identification set $\mathcal{I}$ such that $|\mathcal{I}| \leqslant n / 3$.

This means that knowing the eight edge-labeled trees and $n / 3$ well chosen identifications is enough to encode the floorplan. The cost of recording the trees is exponential, while the cost of writing down $n / 3$ identifications is $n^{n / 3} c^{n}$, hence this proves that $\left|\mathcal{T}_{n}\right| \leqslant n^{n / 3} c^{n}$ and thus completes the proof of Theorem 3 .

Proof of Proposition 19. We begin by showing that identifying the double blocks allows one to reconstruct all identifications. For any pair $i, j$ let us define

$$
\mathcal{I}_{D}^{i, j}=\left\{\left(x_{i}, x_{j}\right): x \in D\right\} \text { and } \mathcal{I}_{D}=\cup_{i, j} \mathcal{I}_{D}^{i, j} .
$$

Lemma 20. For any $i \in\{1,2,3,4\}$ and $j \in\{5,6,7,8\}$, the identification set $\mathcal{I}_{D}^{i, j}$ is strong.

Remark 21. Note that by Proposition 18 knowing $\mathcal{I}_{D}^{i, j}$ is equivalent to knowing $\mathcal{I}_{D}^{i^{\prime}, j^{\prime}}$ for any pair of $i^{\prime}, j^{\prime}$ where $i^{\prime} \in\{1,2,3,4\}$ and $j^{\prime} \in\{5,6,7,8\}$, and also equivalent to knowing $\mathcal{I}_{D}$. Hence we can assume that the whole $\mathcal{I}_{D}$ is given.
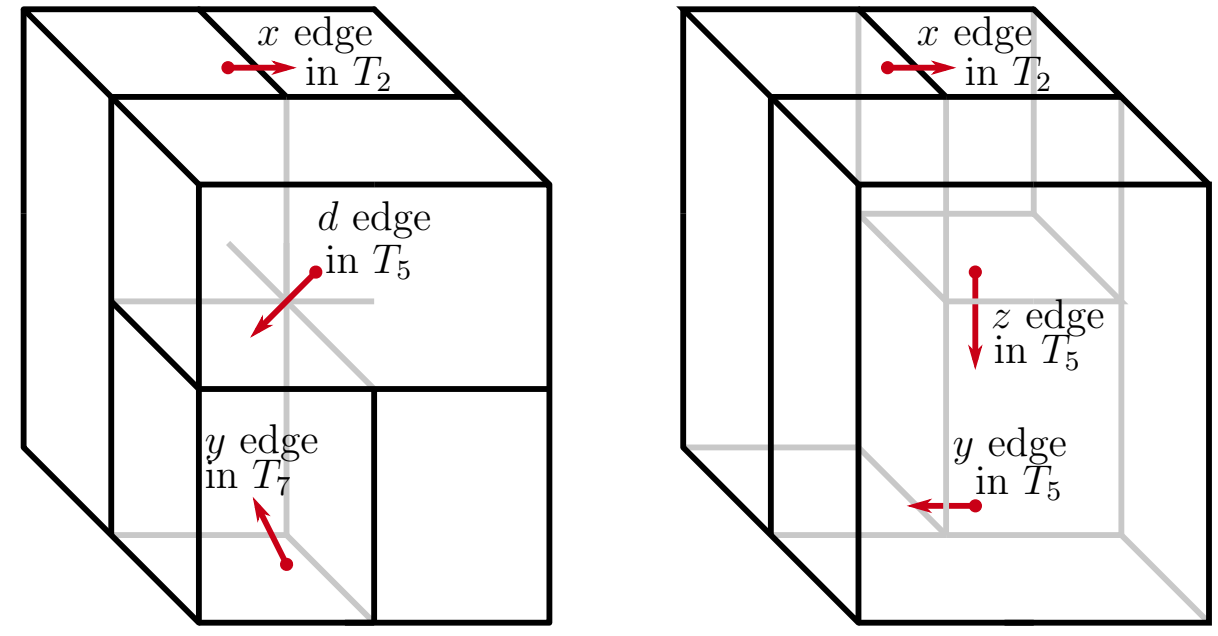

Figure 9: Identification loops

Proof of Lemma 20. Our job is to find $\mathcal{F} \mathcal{I}$ using $\mathcal{I}_{D}^{i, j}$. By Remark 21 and by the symmetric role of the top and bottom layers, it is sufficient to show that we can find the part of $\mathcal{F} \mathcal{I}$ corresponding to blocks in the top layer. By Proposition 18 it is sufficient to find for each block $x \in T$ and each $j \in\{5,6,7,8\}$ the identification of $x_{j}$ with $x_{i}$ for some $i \in\{1,2,3,4\}$. Again, by symmetry, we can concentrate on $j=5$, the other cases follow similarly.

Let's take a look at $\tilde{T}_{5}$. Consider the rooted forest $\tilde{T}_{5}^{\prime}$ obtained by removing all $z$ - and $d$-edges from it. As we have seen in the proof of Proposition 18, the component of $\tilde{T}_{5}^{\prime}$ 
containing the original root corresponds to the blocks of $B \cup D$. All the other components consist of blocks from $T$. Now the crucial (but very easy) observation is that if a top block has an $x$ - or $y$-type parent in $\tilde{T}_{5}$, then it has to have the same type of parent in $\tilde{T}_{1}$, and in fact the parent block has to be the same. Hence all these components of $\tilde{T}_{5}^{\prime}$ are isomorphically there in $\tilde{T}_{1}$. If we can manage to identify the root vertex of each of these components of $\tilde{T}_{5}^{\prime}$ with the appropriate vertex in $\tilde{T}_{1}$, then the rest of the components are automatically identified by the local isomorphisms. Hence to finish the proof of the lemma, all we need is the following: given a vertex $u_{5} \in \tilde{T}_{5}$ whose parent is of type $z$ or $d$, find the corresponding vertex $u_{1} \in \tilde{T}_{1}$.

So let $u_{5}$ be a root vertex of a top component in $\tilde{T}_{5}^{\prime}$. Then $u_{5}$ in $\tilde{T}_{5}$ has either a $d$ - or $z$-type parent. Let us denote this parent by $v_{5} \in \tilde{T}_{5}$ corresponding to some block $v \in F$. First assume it is a diagonal edge, as on the left side of Figure 9 . Since $v_{5}$ is in $B$, by Proposition 18 we can find the corresponding $v_{7} \in \tilde{T}_{7}$. The parent block of this $y_{7}$ in $\tilde{T}_{7}$ has to be necessarily a double block because of how the shape of a diagonal corner looks like. Let us denote this block by $w$. The edge of $y_{7} w_{7}$ in $\tilde{T}_{7}$ can only be an $x$-edge or an $y$-edge. Without loss of generality we may assume the second case. (This is shown on the left side of Figure 9.) Then the local geometry tells us that in $\tilde{T}_{2}$ the parent of $w$ is $u$, the block we started from. Now since $w \in D$ and we know $w_{7}$, we can use the identification set $\mathcal{I}_{D}$ according to Remark 21 to find its counterpart $w_{2} \in \tilde{T}_{2}$. The parent of $w_{2}$ has to be $u_{2}$ in $\tilde{T}_{2}$. In summary, what happened was that we took a vertex $u_{5} \in \tilde{T}_{5}$, looked at a sequence of vertices in various trees, and finally arrived at a vertex of $\tilde{T}_{2}$ that represents the same block. The sequence was

$$
u_{5} \stackrel{d}{\rightarrow} v_{5} \stackrel{B}{\rightarrow} v_{7} \stackrel{y}{\rightarrow} w_{7} \stackrel{\mathcal{I}_{D}}{\rightarrow} w_{2} \stackrel{x}{\rightarrow} u_{2}
$$

where the first, third and last steps involved looking at the parent in the current tree, the second step used Proposition 18 to find a bottom block in another tree, finally the fourth step used $\mathcal{I}_{D}$ to find a double block in another tree. (If the local configuration is the mirror image of what is seen in Figure 9, then $w_{5}$ would be an $x$-type parent of $v_{5}$, then $u$ would be an $y$-type parent of $w$ in $\tilde{T}_{4}$, hence by a similar argument we could find $u_{4} \in \tilde{T}_{4}$.)

Now assume that $v_{5}$ is a $z$-type parent of $u_{5}$, as shown on the right side of Figure 9 . In this case we need to look at the parent of $v_{5}$ in $\tilde{T}_{5}$ still. It is either an $x$ - or a $y$-type parent. Again, without loss of generality, we can assume the second case, as shown in Figure 9. Denoting by $w_{5}$ the parent of $v_{5}$, the local geometry says that $w$, the block represented by this vertex, has to be a double block. Further, in $\tilde{T}_{2}$ the parent of $w$ has to be $u$. We have found $w_{5} \in \tilde{T}_{5}$. Since it is a double block, using $\mathcal{I}_{D}$ according to Remark 21 we can find $w_{2} \in \tilde{T}_{2}$, and looking at its parent we find $u_{2} \in \tilde{T}_{2}$. The sequence now was

$$
u_{5} \stackrel{z}{\rightarrow} v_{5} \stackrel{y}{\rightarrow} w_{5} \stackrel{\mathcal{I}_{D}}{\rightarrow} w_{2} \stackrel{x}{\rightarrow} u_{2} .
$$

(Again, in the mirrored case, if $w_{5}$ is an $x$-type parent of $v_{5}$, then $u_{4}$ would be the $y$-type parent of $w_{4}$ in $\tilde{T}_{4}$, so we could find $u_{4}$ by the same argument.)

Thus in both cases, starting from a vertex in $\tilde{T}_{5}$, we have found its counterpart in $\tilde{T}_{2}$ (or in the mirrored cases in $\tilde{T}_{4}$ ). But by Proposition 18, for any block in the top layer, 
identification between $\tilde{T}_{1}$ and $\tilde{T}_{2}$ (or $\tilde{T}_{4}$ ) is free. So we can finally find $u_{1} \in \tilde{T}_{1}$. This completes the proof of the lemma.

Next, we construct two more strong identification sets. To define these, we need a final bit of notation. Consider a diagonal edge $u_{5} v_{5}$ in $\tilde{T}_{5}$. As we have seen in the proof of Lemma 20 and also in Figure 9, the parent of the block $v$ in $\tilde{T}_{7}$ is a double block $w$. Depending on the type of the $v_{7} w_{7}$ edge, there are two cases. If the $v_{7} w_{7}$ edge is of type $x$, then $u$ is the $y$-parent of $w$ in $\tilde{T}_{4}$. If the $v_{7} w_{7}$ edge is of type $y$, then $u$ is the $x$-parent of $w$ in $\tilde{T}_{2}$. Let us split the diagonal edges of $\tilde{T}_{5}$ into two classes, $d_{4}$ and $d_{2}$, according to these two cases. (The subscript denotes the tree in which $u$ is the parent of $w$.)

We can similarly split the $z$-edges in $\tilde{T}_{5}$ into two classes. In the $z_{2}$ class the child of the $z$-edge in $\tilde{T}_{5}$ is the $x$-parent of a double block in $\tilde{T}_{2}$ while in the $z_{4}$ class the child of the $z$-edge is the $y$-parent of a double block in $\tilde{T}_{4}$. The various classes of edges in $\tilde{T}_{5}$ are shown in Figure 10.

The same classification can be done for $\tilde{T}_{7}$ instead. If $u_{7} v_{7}$ is a diagonal (or $z$-) edge in $\tilde{T}_{7}$ then the parent of $v$ in $\tilde{T}_{5}$ (or in $\tilde{T}_{7}$ ) is a double block $w$, and $u$ is either the $x$-parent of $w$ in $\tilde{T}_{4}$ or the $y$-parent in $\tilde{T}_{2}$. In the first case we call the edge of type $d_{4}$ (or $z_{4}$ respectively) and in the second case type $d_{2}$ (or $z_{2}$ respectively). The local arrangements corresponding to these situations would be mirror reflections of the ones in Figure 10 through the vertical plane adjacent to corners $2,4,6,8$.

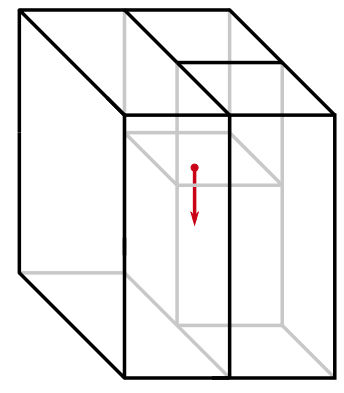

$Z S$-type

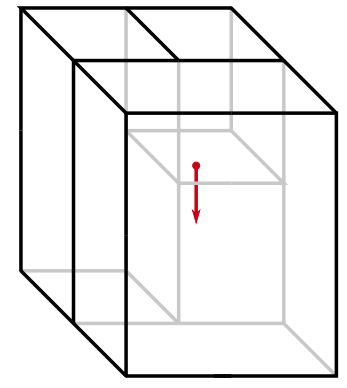

$Z F$-type

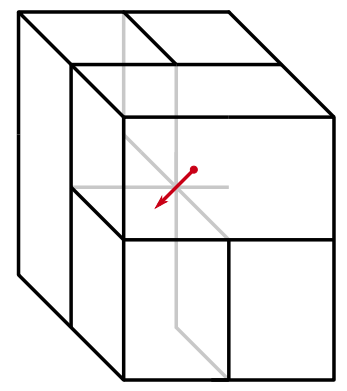

$D S$-type

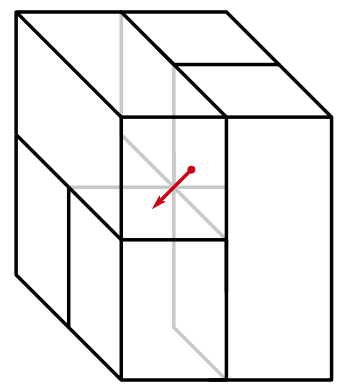

$D F$-type

Figure 10: Refined edge types in $\tilde{T}_{5}$

Now we are ready to define the two identification sets.

$$
\begin{aligned}
& \mathcal{I}_{T}^{4}=\left\{\left(u_{5}, u_{4}\right): u_{5} \in \tilde{T}_{5} \text { is a } d_{4} \text { or } z_{4} \text { child }\right\} \cup\left\{\left(u_{7}, u_{4}\right): u_{7} \in \tilde{T}_{7} \text { is a } d_{4} \text { or } z_{4} \text { child }\right\}, \\
& \mathcal{I}_{T}^{2}=\left\{\left(u_{5}, u_{2}\right): u_{5} \in \tilde{T}_{5} \text { is a } d_{2} \text { or } z_{2} \text { child }\right\} \cup\left\{\left(u_{7}, u_{2}\right): u_{7} \in \tilde{T}_{7} \text { is a } d_{2} \text { or } z_{2} \text { child }\right\} .
\end{aligned}
$$

Lemma 22. The identification sets $\mathcal{I}_{T}^{2}$ and $\mathcal{I}_{T}^{4}$ are both strong.

Proof. Since the definitions of the two sets are symmetric, it suffices to show that $\mathcal{I}_{T}^{2}$ is strong. In the light of Lemma 20 if we can show that all the vertices representing double blocks in $\tilde{T}_{2}$ can be identified with their counterparts in $\tilde{T}_{6}$ then we are done. 
We do this identification recursively: if a parent of a double block $w_{2} \in \tilde{T}_{2}$ is an other double block $u_{2} \in \tilde{T}_{2}$, then, since the whole floorplan has only two layers, the parent of $w_{6} \in \tilde{T}_{6}$ has to be the $u_{6}$, and the two parents have to have the same type. Hence if we could identify $u_{2}$ with $u_{6}$, and we find that the parent of $w_{2}$ is $w_{6}$ of type $x$, then by looking at the $x$-child of $u_{6}$ we can find $u_{2}$. (Similarly for $y$-type parents.) Thus we only have to worry about double blocks $w$ for which the parent of $w_{2} \in \tilde{T}_{2}$ is a single block (hence of type $T$ ) $u_{2} \in \tilde{T}_{2}$.

Suppose first that $u_{2}$ is an $x$-parent of $w_{2}$. Then the local arrangement has to look like as in Figure 10. Thus the parent $v$ of the top block $u$ in $\tilde{T}_{5}$ has to be a $z$ - or $d$-parent, furthermore it can only be of type $z_{2}$ or $d_{2}$. This means that $\left(u_{5}, u_{2}\right) \in \mathcal{I}_{T}^{2}$. We can use this to trace an identification loop as before:

$$
w_{2} \stackrel{x}{\rightarrow} u_{2} \stackrel{\mathcal{I}_{T}^{2}}{\rightarrow} u_{5} \stackrel{d_{2}}{\rightarrow} v_{5} \stackrel{B}{\rightarrow} v_{7} \stackrel{y}{\rightarrow} w_{7} \stackrel{B}{\rightarrow} w_{6},
$$

or

$$
w_{2} \stackrel{x}{\rightarrow} u_{2} \stackrel{\mathcal{I}_{T}^{2}}{\rightarrow} u_{5} \stackrel{z_{2}}{\rightarrow} v_{5} \stackrel{x}{\rightarrow} w_{5} \stackrel{B}{\rightarrow} w_{6},
$$

depending on type of the $u_{5} v_{5}$ edge. In other words, starting from $w_{2}$, and looking at parents or jumping between trees using the known identifications, we find its counterpart $w_{6} \in \tilde{T}_{6}$.

Similarly, if $u_{2}$ is an $y$-parent of $w_{2}$. Then the local arrangement has to look like the mirror image of those in Figure 10. Thus the parent $v$ of the top block $u$ in $\tilde{T}_{7}$ has to be a $z$ - or $d$-parent, furthermore it can only be of type $z_{2}$ or $d_{2}$. The argument is then the same as above. This completes the proof of the lemma.

Now it is very easy to finish proving the proposition. Clearly, $\left|\mathcal{I}_{D}^{i, j}\right|=|D|$. On the other hand, observe, that $\mathcal{I}_{T}^{2} \cup \mathcal{I}_{T}^{4}$ has exactly one identification written down for each vertex having a $z$ - or a $d$-parent in $\tilde{T}_{5}$, and one identification for each vertex having a $z$ or $d$-parent in $\tilde{T}_{7}$. But only top blocks can have $z$ - or $d$-parents, hence $\left|\mathcal{I}_{T}^{2} \cup \mathcal{I}_{T}^{4}\right| \leqslant 2|T|$, and so one of them has to be at most of size $|T|$. Finally one could repeat the whole argument of Lemma 22 with identifications sets $\mathcal{I}_{B}^{6}$ and $\mathcal{I}_{B}^{8}$ defined entirely analogously, reversing top and bottom everywhere. Then one of these two strong identification sets have to be of size at most $|B|$.

So we have found three identification sets of size at most $|D|,|T|$, and $|B|$ respectively. Since $|D|+|T|+|B|=n$, one of these have to be of size at most $n / 3$, proving the proposition, completing the proof of Theorem 3.

\section{References}

[1] E. Ackerman. Counting problems for geometric structures: rectangulations, floorplans, and quasi-planar graphs. PhD Thesis, 2006, Technion, Haifa, Israel.

[2] E. Ackerman, G. Barequet, and R. Y. Pinter. A bijection between permutations and floorplans, and its applications. Discrete Applied Math., 154(12):1674-1684, 2006. 
[3] R. Fischbach, J. Leinig, J. Knechtel, Investigating modern layout representations for improved 3D design automation, Proceedings of 21st Great lakes symposium on VLSI, pp. 337-342, 2011

[4] P. Garrou, C. Bower, and P. Ramm (eds), Handbook of 3D Integration: Technology and Applications of 3D Integrated Circuits, Vols. 1, 2, Wiley-VCH, 2008.

[5] Y. Ma, X. Hong, S. Dong, C-K. Cheng, 3D CBL: an efficient algorithm for general 3D packing problems., Proceedings of 48th Midwest Symposium on Circuits and Systems, pp. 1079-1082, 2005

[6] H. Ohta, T. Yamada, C. Kodama, and K. Fujiyosi, The O-Sequence: Representation of 3D-floorplan dissected by rectangular walls, Prime '06, pp. 317-320, 2006.

[7] B. Yao, H. Chen, C-K. Cheng, and R. Graham. Floorplan representations: complexity and connections. ACM Trans. on Design Automation of Electronic Systems, pp. 5580, Jan. 2003

[8] R. Wang, E.F.Y. Young, and C-K. Cheng. Representing topological structures for 3-D floorplanning. ICCCAS, pp.1098-1102, July 2009 